\title{
Complicaciones postquirúrgicas de la malformación de Chiari tipo I: duroplastia y fístula de líquido cefalorraquídeo
}

\author{
J.M. Saceda-Gutiérrez; A. Isla-Guerrero; F. Álvarez-Ruíz; C. Odene-Cantero; B. Hernández-García y T.M. Márquez- \\ Pérez
}

Servicio de Neurocirugía. Hospital Universitario La Paz. Madrid.

Resumen

El tratamiento quirúrgico de la malformación de Chiari tipo I consiste en realizar una descompresión ósea suboccipital. Está descrito que pueden producirse complicaciones derivadas de la cirugía, sobre todo si se realiza apertura dural y posterior cierre con injerto (duroplastia). Una de las más importantes por su difícil manejo, es la fístula de líquido cefalorraquídeo en la zona quirúrgica.

Objetivo. Llevar a cabo una revisión postquirúrgica, donde analizaremos la evolución de los pacientes y la correlación de las complicaciones respecto al tipo de plastia dural usada.

Material y métodos. Se ha realizado un estudio retrospectivo de los pacientes intervenidos en nuestro centro entre el año 1997 y 2008, ambos inclusive, recogiendo un total de 36 pacientes. A todos ellos se les realizó un estudio pre y postoperatorio con resonancia magnética craneoespinal, así como un examen neurológico antes y después de la intervención. La intervención quirúrgica consistió en una descompresión ósea suboccipital, junto con la extirpación del arco posterior de C1 o C2 (dependiendo del descenso amigdalar), y duroplastia con injerto autólogo (pericráneo) o con injerto heterólogo (Gore-tex).

Resultados. Tras un seguimiento medio de 2 años obtuvimos los siguientes resultados clínicos: excelente (55\%), si hubo gran mejoría clínica; bueno (29\%), si hubo leve mejoría; y malo (16\%), si no hubo mejoría o hubo empeoramiento. En los 30 pacientes a los que se les realizó duroplastia (18 plastia artificial, 12 plastia autóloga de pericráneo occipital), aparecieron 6 casos de fuga de líquido cefalorraquídeo, no existiendo asociación significativa entre el tipo de plastia dural y la presencia de fístula.

Conclusiones. Los síntomas que mejoran en mayor medida en los pacientes intervenidos en nuestro centro son la cefalea, el dolor cervical y el mareo. A

Recibido: 25-05-10. Aceptado: 9-07-10 pesar de existir mayor número de fístulas de líquido cefalorraquídeo en el grupo de pacientes con plastia artificial respecto al grupo con plastia de pericráneo, no es un hallazgo significativo.

PALABRAS CLAVE: Malformación de Chiari I. Duroplastia. Fístula de LCR. Pseudomeningocele. Siringomielia.

Postoperative complications in Chiari I malformation: duroplasty and cerebrospinal fluid leak

Summary

The surgical treatment of Chiari I malformation is to carry out a suboccipital decompression. It is described that postoperative complications may occur, especially if the dura is open and closed using a graft (duraplasty). Among them, one of the most important events due to its difficult handling is cerebrospinal fluid leak through the suture line.

Objective. To conduct a postoperative review to analyze the outcome of the patients and the occurrence of complications depending on the dural plasty used.

Material and methods. A retrospective study was carried out between 1997 and 2008, both inclusive, where we assessed 36 patients. All of them were studied with preoperative and postoperative craneoespinal magnetic resonance, and by a thorough clinical examination performed before and after the surgery. The surgical procedure consisted of suboccipital decompression and resection of the posterior arch of $\mathrm{C} 1$ or $\mathrm{C} 1$ and C2 (depending on the extent of the caudal displacement of the tonsils), followed by duraplasty using either an autologous graft (pericranium) or a synthetic graft (Gore-tex).

Results. After a mean follow-up of 2 years, the clinical results were: excellent $(55 \%)$, if there was a great

Abreviaturas. RM: resonancia magnética. LCR: líquido cefalorraquídeo. 
clinical improvement; good $(\mathbf{2 9 \%})$, if there was slight improvement; and bad (16\%), if there was no improvement or there was worsening. In the 30 patients given a duraplasty (18 with an artificial graft, 12 with an autologous pericranium graft), 6 cases of cerebrospinal fluid leak appeared, although no significant association between the type of dural plasty and the presence of leak was observed.

Conclusions: The best results were obtained for headaches, cervical pain and dizziness. Despite the fact that there were more cases of cerebrospinal fluid leak in patients receiving an artificial graft compared to patients with pericranium graft, there was no significant difference.

KEY WORD: Chari I malformation. Duroplasty. CSF leak. Pseudomeningocele. Syringomyelia.

\section{Introducción}

En 1891, Hans Chiari presentó sus primeros hallazgos patológicos encontrados en las autopsias de pacientes con hidrocefalia y ectopia cerebelosa, acuñando el concepto de malformación de Chiari tipo I, que se caracterizaba por la "elongación de las amígdalas cerebelosas y división de la parte inferior de los lóbulos del cerebelo descendiendo ambos hacia el canal raquídeo"14. En el estudio de resonancia magnética, la malformación de Chiari tipo I se define por un descenso de las amígdalas cerebelosas por debajo del foramen magno de al menos 5 milímetros $^{1}$. Sin embargo, el criterio radiológico aislado no es suficiente, ya que con esta condición el $0,8 \%$ de los pacientes están asintomáticos ${ }^{11,18}$. Por tanto, el hallazgo de desplazamiento amigdalar aislado es de limitada utilidad, y se debe tener en cuenta la sintomatología del paciente.

Existen varias teorías para explicar la génesis del desplazamiento amigdalar, siendo la más aceptada, la desproporción volumétrica de las estructuras neurales (troncoencéfalo y cerebelo), respecto al tamaño craneal de la fosa posterior ${ }^{19}$.

La malformación de Chiari tipo I puede asociar la presencia de siringomielia entre el 50 al $70 \%$ de los casos 9 . Para explicar la génesis de la siringomielia han surgido muchas hipótesis, pero actualmente la más aceptada es la teoría del "efecto pistón" de Oldfield ${ }^{27}$, que se basa en que el aumento de presión intracraneal durante la sístole cardiaca provoca una onda de presión en el espacio subaracnoideo perimedular, debido a las adherencias a nivel del foramen magno, que hace filtrase el líquido cefalorraquídeo (LCR) a través de los espacios perivasculares e intersticiales de la superficie medular ${ }^{17,23}$.

El tratamiento quirúrgico de la malformación de
Chiari tiene una serie de objetivos principales: 1) corregir el gradiente de presión cráneo-espinal; 2) aumentar el volumen total de la fosa posterior; 3) restablecer el flujo de LCR a través de la cisterna magna; 4) en caso de que exista, disminuir el tamaño de la cavidad siringomiélica; y 5) aliviar la sintomatología debida a la compresión del tronco cerebral ${ }^{7,33}$.

Los pacientes pueden estar asintomáticos ${ }^{22}$. La cefalea y la sensación de mareo son los síntomas más frecuentes, y generalmente empeoran con la maniobra de Valsalva. Puede aparecer afectación motora, sensitiva, cerebelosa y de pares craneales bajos, empeorando el pronóstico en tal caso. La presencia de déficit neurológico o de sintomatología evolutiva, son criterios de intervención quirúrgica. La presencia de siringomielia en pacientes asintomáticos, es motivo de controversia en la bibliografía, existiendo autores que abogan por la intervención quirúrgica, sobre todo si alcanza un tamaño de $8 \mathrm{~mm}$ de diámetro o en caso de asociar escoliosis progresiva ${ }^{31}$.

Las primeras técnicas quirúrgicas para tratar esta patología fueron: el taponamiento del obex, la colocación de un stent en el cuarto ventrículo, la craniectomía amplia de fosa posterior, y la laminectomía multinivel. Actualmente, el abordaje quirúrgico habitual es la descompresión ósea suboccipital (aproximadamente de $3 \times 3 \mathrm{~cm}$ ) 34 , asociado con laminectomía del arco posterior de $\mathrm{C} 1$ o $\mathrm{C} 2$ (dependiendo del grado de desplazamiento caudal de las amígdalas) ${ }^{3,24}$. Junto a la resección ósea, es importante la apertura de la banda fibrosa occipito-atloidea que se forma a nivel del foramen magno, y que presenta características histológicas específicas en este tipo de pacientes ${ }^{26}$. Para conseguir mayor grado de descompresión se puede abrir la duramadre de la fosa posterior, sólo la capa externa mediante incisiones ${ }^{15}$; o lo que es más habitual, abriendo las dos capas de la duramadre, generalmente en forma de "Y" para posteriormente realizar una plastia dural con pericráneo ${ }^{30,32}$, fascia lata, duramadre de cadáver ${ }^{35}$ o sustitutivos durales artificiales como el gore-tex ${ }^{29}$. Algunos autores proponen la coagulación ${ }^{38}$ o resección uni o bilateral de las amígdalas cerebelosas para conseguir una óptima descompresión en la unión cráneo-cervical ${ }^{12,13}$.

Además de los problemas habituales de cualquier cirugía, como la infección o la hemorragia, existen complicaciones más específicas dependiendo del tipo de intervención llevada a cabo.

Está descrito en la bibliografía que en pacientes intervenidos mediante duroplastia pueden aparecer diversas complicaciones, entre las que destacan: meningitis aséptica, reacción inflamatoria asociada a la plastia dural, hidrocefalia, pseudomeningocele y fístula de $\mathrm{LCR}^{36}$. Por contra, la descompresión de la fosa posterior sin duroplastia puede presentar como principal complicación la inadecuada descompresión ósea con el riesgo de la reaparición de la 
sintomatología ${ }^{12}$.

La fístula de LCR es una de las complicaciones más temibles de los abordajes a la fosa posterior por su difícil manejo, que en ocasiones suele requerir una nueva intervención quirúrgica para solucionarla. Debido a esto, existen multitud de artículos en la bibliografía que analizan las ventajas e inconvenientes de realizar duroplastia o no: para algunos autores está justificado no abrir la dura por el riesgo postoperatorio de fístula que existe, y porque no parece existir una clara diferencia en la sintomatología postquirúrgica del paciente, respecto a los que se les realiza además duraplastia; por otra parte hay autores que asumen el riesgo de aparición de fístula, porque la esencia de la descompresión de la fosa posterior se basa en apertura dural, y porque en sus series sí que encuentran diferencias clínicas postoperatorias en ambos grupos de pacientes ${ }^{8}$.

El objetivo del presente trabajo es realizar una revisión de los resultados postquirúrgicos donde analizaremos la evolución de los pacientes, y la correlación de las complicaciones respecto al tratamiento quirúrgico realizado.

\section{Material y métodos}

Se ha realizado un estudio retrospectivo de los pacientes intervenidos de malformación de Chiari I en el Hospital de La Paz entre el año 1997 y 2008, ambos inclusive. Recogiendo un total de 36 pacientes, 26 mujeres y 10 hombres, con edades comprendidas entre los 19 y los 78 años (edad media 43 años). 16 pacientes asociaban cavidad siringomiélica. A todos ellos, se les realizó estudio de imagen con resonancia magnética (RM) craneoespinal preoperatoria y otra entre 3-6 meses tras la intervención. Recogimos los exámenes neurológicos pre y postquirúrgicos, de acuerdo con los siguientes parámetros: manifestaciones medulares (alteraciones sensitivas, alteraciones motoras y trastornos en el control de esfínteres), manifestaciones cerebelosas (nistagmo, ataxia, disartria, Romberg), manifestaciones de tronco cerebral (sordera, disfagia, disfonía, disestesia trigeminal, vértigo) y otras alteraciones clínicas frecuentes como cefalea, cervicalgia, cervicobraquialgia o mareo.

\section{Técnica quirúrgica}

Todos los pacientes fueron intervenidos en posición de decúbito prono con la cabeza fijada mediante el cabezal de Mayfield. Se realizó una pequeña craniectomía suboccipital con extirpación del arco posterior de C1, y a veces de C2, en función del descenso amigdalar. Se resecó el manguito fibroso que se forma en la zona del foramen magno y que comprime la dura en la zona posterior. En caso de realizar apertura dural, se hizo una incisión en forma de " $Y$ " intentando preservar la aracnoides. Posteriormente la dura se cerró mediante una plastia autóloga de pericráneo occipital o una plastia heteróloga de Gore-Tex.

\section{Método estadístico}

Intentamos calcular si existía relación estadística entre: el uso de plastia dural autóloga, o el uso de plastia artificial, con la aparición de fístula de LCR. Para comparar estas variables cualitativas independientes se ha usado el test exacto de Fisher. Como nivel de significación estadística se asume una $\mathrm{p}<0,05$. Los cálculos se realizaron con el programa estadístico SPSS 11.0 para Windows.

\section{Resultados}

\section{Clínicos}

Los síntomas más frecuentes de presentación fueron la cefalea y/o dolor cervical $(72 \%)$, el mareo (52\%), y las parestesias en miembros superiores (72\%). Los signos más frecuentes fueron las alteraciones cerebelosas $(58 \%)$, y el déficit motor (31\%) que afectaba principalmente a las extremidades superiores.

Tras un seguimiento mínimo de 6 meses (media de 24 meses) obtuvimos los siguientes resultados (tabla $1 \mathrm{y}$ $2)$ : excelente $(20 / 36,55 \%)$, si hubo gran mejoría clínica; bueno $(10 / 36,29 \%)$, si hubo leve mejoría; malo (6/36, $16 \%$ ), si no hubo mejoría o hubo empeoramiento.

Tabla 1

Signos y síntomas pre y postoperatorios

\begin{tabular}{|l|c|c|c|c|c|c|c|c|}
\hline Clínica & $\begin{array}{l}\text { Cefalea } \\
\text { Dolor } \\
\text { cervical }\end{array}$ & $\begin{array}{c}\text { Alteración } \\
\text { Sensitiva }\end{array}$ & $\begin{array}{l}\text { Alteración } \\
\text { Crebelo }\end{array}$ & Mareo & $\begin{array}{c}\text { Alteración } \\
\text { motora }\end{array}$ & Nistagmo & $\begin{array}{l}\text { Alt. } \\
\text { pares } \\
\text { bajos }\end{array}$ & Otros \\
\hline Preop & 26 & 26 & 21 & 19 & 11 & 7 & 3 & 3 \\
\hline Postop & 11 & 15 & 10 & 1 & 2 & 4 & 0 & 0 \\
\hline
\end{tabular}

Preop: preoperatorio. Postop: postoperatorio. 
Tabla 2

Diagrama representando la evolución clínica

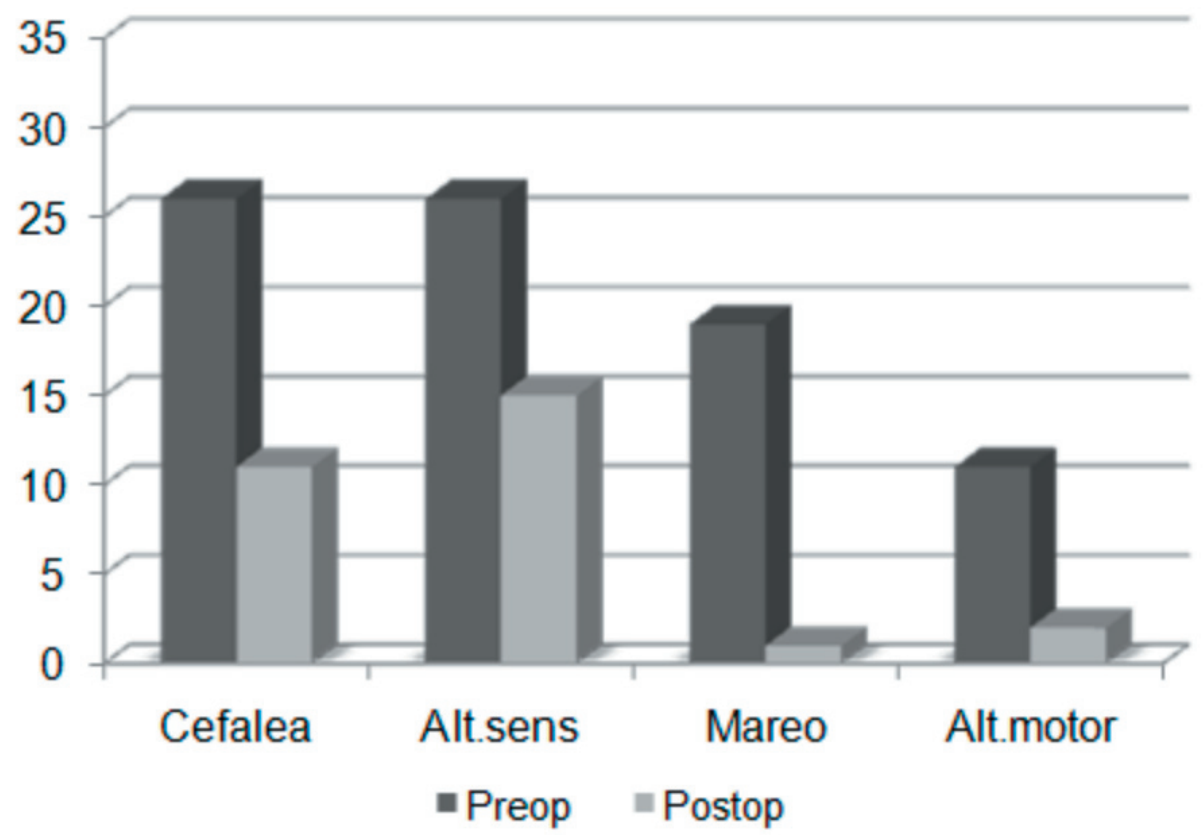

\section{Datos quirúrgicos}

De los 36 pacientes analizados: A 30 de ellos se les realizó duroplastia (18 con Gore-Tex, 12 con pericráneo occipital), y en 6 casos sólo se realizó descompresión ósea.

\section{Complicaciones postoperatorias}

Encontramos un total de 12 complicaciones (todas ellas ocurrieron en pacientes a los que se les realizó duroplastia): 6 casos de fuga de LCR (3 colecciones subcutáneas y 3 fístulas externas de LCR); 3 casos de meningitis (2 asépticas y 1 bacteriana); 2 casos de infección de herida quirúrgica; y 1 caso de muerte por hematoma de fosa posterior.

Analizamos los casos de fuga de LCR (Figura 1), e intentamos encontrar relación entre la aparición de salida de LCR y el tipo de plastia usada durante la reconstrucción dural. Para ello, analizamos los 30 pacientes a los que se les realizó duroplastia y se les dividió en dos grupos: uno de plastia artificial (Gore-Tex), y otro de plastia autóloga (pericráneo occipital). De esta forma se buscó la asociación estadística entre ambas variables.

Para realizar el estudio estadístico se utilizó el test exacto de Fisher, donde se obtuvo una $\mathrm{p}=0,358$. Este valor descarta en nuestra muestra la asociación estadísticamente significativa entre el uso de un tipo de una plastia dural $\mathrm{u}$ otra, con la aparición de fuga de LCR (tabla 3).

\section{Discusión}

\section{Datos clínicos}

Los hallazgos clínicos encontrados en nuestra serie son similares a los encontrados en la bibliografía ${ }^{6,9,16}$. La cefalea ha sido el síntoma principal, y algunos de estos pacientes fueron tratados previamente de forma incorrecta como migraña compleja. Junto a la cefalea, el dolor cervical irradiado a los miembros superiores fue otro síntoma que se encontró en muchos pacientes, y generalmente empeoraba con maniobras de Valsalva. La cefalea, el dolor cervical y el mareo han sido en nuestra serie los síntomas que mejor han respondido a la intervención quirúrgica, hecho en concordancia con otras publicaciones ${ }^{36}$.

El déficit motor afectaba a 11 pacientes (31\%), el grado de afectación y el porcentaje es algo menor que el encon-

Tabla 3

Datos usados para calcular la asociación estadística entre las variables

\begin{tabular}{|l|c|c|c|}
\hline Plastia & Fístula & No fístula & Total \\
\hline Artificial & 5 & 13 & 18 \\
\hline Autóloga & 1 & 11 & 12 \\
\hline & 6 & 24 & 30 \\
\hline
\end{tabular}



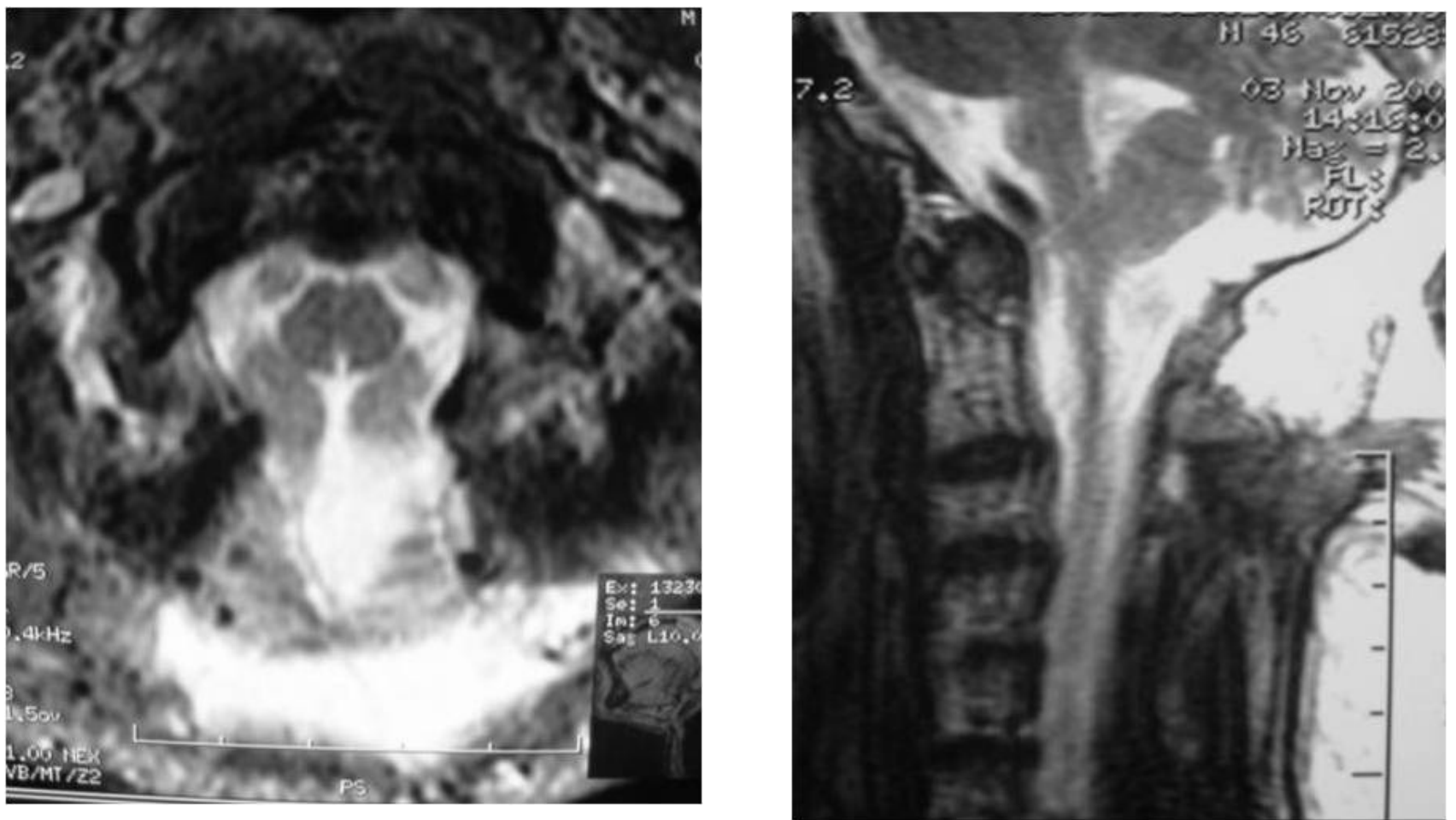

Figura 1. Imágenes sagital y axial de RM en secuencia T2, que muestra una hiperseñal compatible con presencia de LCR en la zona de la musculatura cervical posterior.

trado en otras series antiguas, debido en parte a la mejora en las técnicas de neuroimagen en la última década ${ }^{29}$. En nuestra serie, 9/11 (81\%) mejoraron, esto difiere de algunas publicaciones en las que se cita que una vez se establece la afectación motora no se produce mejoría postoperatoria ${ }^{4,20}$.

La afectación sensitiva, en forma de disestesias y parestesias, ha sido el problema neurológico que menos ha respondido a la intervención quirúrgica, solo 11/26 (42\%) mejoraron, porcentaje inferior al encontrado en otras series publicadas $^{19}$.

\section{Complicaciones postoperatorias}

Se han descrito una serie de complicaciones que pueden ocurrir de forma precoz o tardía tras la cirugía: hemorragia del lecho quirúrgico, meningitis aséptica, reacción inflamatoria al parche dural, fístula de LCR y pseudomeningocele, hidrocefalia, infección de herida, y ptosis cerebelosa tras craniectomía extensa ${ }^{25}$.

La meningitis aséptica no es una complicación per se de la cirugía. La irritación del cuarto ventrículo y las estructuras que lo rodean causa síntomas como cefalea, dolor cervical, febrícula, náuseas y vómitos. Generalmente el cuadro responde con tratamiento conservador mediante analgésicos y antiinflamatorios ${ }^{21}$. En nuestra serie hemos recogido dos casos que han cursado con una clínica y una respuesta terapéutica parecida a lo encontrado en la bibliografía.
La fístula de LCR y el pseudomeningocele pueden presentarse como complicaciones precoces o tardías ${ }^{37}$. El cierre inadecuado de la dura o de la fascia muscular cervical posterior puede permitir la salida de LCR desde la fosa posterior. Además, la hidrocefalia postoperatoria es una causa común de fuga de líquido con la presencia o no de pseudomeningocele ${ }^{11}$, debiéndose realizar una RM y un fondo de ojo, para descartar ventriculomegalia y papiledema respectivamente. En caso de existir alguno de éstos puede ser necesaria la colocación de un drenaje ventricular externo, y posteriormente valorar la posible implantación de una derivación ventrículo peritoneal. La presencia de un pseudomeningocele requiere reparación quirúrgica si se asocia a fístula de LCR, papiledema, siringomielia ${ }^{28} \mathrm{o}$ si el defecto estético es importante.

La elección de un tipo de plastia puede condicionar la recurrencia o el empeoramiento de la sintomatología preoperatoria ${ }^{10}$, este hecho se ha descrito especialmente con el uso de plastias heterólogas ${ }^{30}$. El cierre de la dura es un paso crítico para evitar la aparición de complicaciones, aunque no existen estudios al respecto que justifiquen que un tipo de sutura sea mejor que otra. Respecto al uso de un tipo de plastia en concreto para disminuir la tasa de fugas de LCR, tampoco hay estudios previos concluyentes que apoyen o contradigan los hallazgos de nuestra serie.

$\mathrm{Si}$ apareciese la salida de líquido, es necesaria: una buena limpieza de la herida, aplicar un vendaje compre- 
sivo en la zona, valorar la posibilidad de suturar de nuevo alguna parte de herida, y si no fuese suficiente con todo esto, se colocaría un drenaje lumbar ${ }^{21}$. En nuestra serie encontramos 6 casos de fuga de LCR: 3 pacientes con acumulo subcutáneo (1 mejoró con vendaje compresivo y 2 con vendaje compresivo mas drenaje lumbar 3-5 días); 3 pacientes con fístula externa (1 mejoró con vendaje mas drenaje y sutura de la herida, 1 mejoró con retirada de plastia artificial e implantación de fascia lata, y 1 mejoró con refuerzo de cierre de la plastia autóloga previa). Al igual que otras series halladas en la bibliografía ${ }^{2}$, todas las complicaciones encontradas en nuestra muestra aparecieron en los casos en los que se realizó duroplastia. A pesar de que la tasa de complicaciones es mayor en los casos que se abre la dura, algunos autores asumen la aparición de estos problemas postoperatorios, porque encuentran una clara diferencia en la mejoría clínica tras la cirugía cuando se realiza duroplastia ${ }^{5}$.

\section{Conclusiones}

Los síntomas que mejoran en mayor medida en los pacientes intervenidos en nuestro centro son la cefalea, el dolor cervical y el mareo; y el signo que más se beneficia de la cirugía es el déficit motor. Por el contrario el signo que menos se ha corregido ha sido el déficit sensitivo.

En nuestra muestra de pacientes parece existir un mayor número de complicaciones postoperatorias en el grupo tratado con duroplastia. Aunque exista mayor número de fístulas de líquido cefalorraquídeo en el grupo de pacientes con plastia artificial respecto al grupo con plastia de pericráneo, este hallazgo no fue estadísticamente significativo.

Generalmente los casos de fístula de LCR, bien en forma de colección subcutánea o en forma de fístula externa, responden adecuadamente con las medidas conservadoras sin necesidad de reintervención.

\section{Bibliografia}

1. Aboulezz, A.O., Sartor, K., Geyer C.A.: Position of cerebellar tonsils in the normal population and in patients with Chiari malformation: a quantitative approach to MR imaging. J Comput Assist Tomogr 1985; 9: 1033-1036.

2. Alamar, M., Teixidor, P., Colet, S., et al.: Comparación del tratamiento de la malformación de Chiari tipo I mediante craniectomía suboccipital y resección del arco posterior de C1 con o sin duroplastia. Neurocirugía 2008; 19: 233-241.

3. Alden, T.D., Ojemann, J.G., Park, T.S.: Surgical treatment of Chiari I malformation: indications and approaches. Neurosurg Focus 2001; 11: 1-5.

4. Alvarez-Betancourt, L.A., García Rentaría, J.A., López Ortega, S.J., et al.: Malformación de Chiari tipo I: evolución postoperatoria a 2 años. Análisis de 10 casos. Neurocirugía 2005; 16: 34-38.

5. Alzate, J., Kothbauer, K., Jallo, G.: Treatment of Chiari type I malformation in patients with and without syringomyelia: a consecutive series of 66 cases. Neurosurg Focus 2001; 11: 1-9.

6. Badie, B., Mendoza, D., Batzdorf, U.: Posterior fossa volume and response to suboccipital decompression in patients with Chiari I malformation. Neurosurgery 1995; 37: 214-218.

7. Batzdorf, U.: Chiari I malformation with syringomyelia. J Neurosurg 1988; 68: 726-730.

8. Caldarelli, M.D., Novegno, F., Massimi, L., Romani, R., Tamburrini, G., Di Rocco.: The role of limited posterior fossa craniectomy in the surgical treatment of Chiari malformation Type I: experience with a pediatric series. J Neurosurg 2007; 106: 187-195.

9. Depreitere, B., Van Calenbergh, F., Van Loon, J.: Posterior fossa decompression in syringomyelia associated with a Chiari malformation: a retrospective analysis of 22 patients. Clin Neurol Neurosurg 2000; 102: 91-96.

10. Dyste, G.N., Menezes, A.H., VanGilder, J.C.: Symptomatic Chiari malformations. An analysis of presentation, management, and long-term outcome. J Neurosurg 1989; 71: 159-168.

11. Elton, S., Tubbs, R.S., Wellons, J.C.: Acute hydrocephalus following a Chiari I decompression. Pediatr Neurosurg 2002; 36: 101-104.

12. Fessler, R.G., Rhoton, A.L. Jr.: Chiari malformation and syringomyelia, in Little JR, Awad IA (eds): Preoperative Neurosurgery. Baltimore: Williams \& Wilkins, 1992; pp. 113127.

13. Fisher, E.: Posterior fossa decompression for Chiari I deformity, including resection of the cerebellar tonsils. Childs Nerv Syst 1995; 11: 625-629.

14. Ghassan, K.B.: Definition of the adult Chiari malformation: a brief historical overview. Neurosurg Focus 2001; 11: 1-8.

15. Hanieh, A., Sutherland, A., Foster, B., Cundy, P.: Syringomyelia in children with primary scoliosis. Childs Nerv Syst 2000; 16: 200-202.

16. Hida, K., Iwasaki, Y., Koyanagi, I.: Surgical indication and results of foramen magnum decompression versus syringosubarachnoid shunting for syringomyelia associated with Chiari I malformation. Neurosurgery 1995; 37: 673-679.

17. Klekamp, J., Batzdorf, U., Samii, M., Bothe, H.W.: The surgical treatment of Chiari I malformation. Acta Neurochir (Wien) 1996; 138: 788-801.

18. Koehler, P.J.: Chiari's description of cerebellar ectopy (1891). With a summary of Cleland's and Arnold's contributions and some early observations on neural-tube defects. J Neurosurg 1991; 75: 823-826.

19. Krieger, M.D., McComb, J.G., Levy, M.L.: Toward a simpler surgical management of Chiari I malformation in a 
pediatric population. Pediatr Neurosurg 1999; 30: 113-121.

20. Logue, V., Edwards, M.R.: Syringomyelia and its surgical treatment- an analysis of 75 patients. J Neurol Neurosurg Psychiatry 1981; 44: 273-284.

21. Mazzola, C., Arno, H.. F.: Revision surgery for Chiari malformation surgery. Neurosurg Focus 2003; 15: 1-8.

22. Meadows, J., Kraut, M., Guarnieri, M., et al.: Asymptomatic Chiari Type I malformations identified on magnetic resonance imaging. J Neurosurg 2000; 92: 920-926.

23. Menezes, A.H.: Primary craniovertebral anomalies and the hindbrain herniation syndrome (Chiari I): data base analysis. Pediatr Neurosurg 1995; 23: 260-269.

24. Milhorat, T.H., Chou, M.W.: Trinidad, E.M. Chiari I malformation redefined: clinical and radiographic findings for 364 symptomatic patients. Neurosurgery 1999; 44: 10051017.

25. Munshi, I., Frim, D., Stiene-Reyes, R.: Effects of posterior fossa decompression with or without duroplasty on Chiari malformation-assiciated hydromyelia. Neurosurgery 2000; 46: 1384-1390.

26. Nakamura, N., Iwasaki, Y., Hida, K., et al.: Dural band pathology in syringomyelia with Chiari type I malformation. Neuropathology 2000; 20: 38-43.

27. Oldfield, E.H., Muraszko, K., Shawker, T.H.: Pathophysiology of syringomyelia associated with Chiari I malformation of the cerebellar tonsils. Implications for diagnosis and treatment. J Neurosurg 1994; 80: 3-15.

28. Pare, L.S., Batzdorf, U.: Syringomyelia persistence after Chiari decompression as a result of pseudomeningocele formation: implications for syrinx pathogenesis: report of three cases. Neurosurgery 1998; 43 : 945-948.

29. Paul, K.S., Lye, R.H., Strang, F.A.: Arnold-Chiari malformation. Review of 71 cases. J Neurosurg 1983; 58: 183-187.

30. Rosen, D.S., Wollman, R., Frim, D.M.: Recurrence of symptoms after Chiari decompression and duraplasty with nonautologous graft material. Pediatr Neurosurg 2003; 38 : 186-190.

31. Sahuquillo, J., Rubio, E., Poca, M.A., Rovira, A., Rodrí-

\section{Comentario al trabajo Complicaciones postquirúr- gicas de la malformación de Chiari tipo I: duroplastia y fístula de liquido cefalorraquídeo de Saceda Gutiérrez y cols.}

Los autores describen sus resultados postoperatorios de 36 pacientes operados de malformación de Chiari-1, con especial énfasis en las complicaciones asociadas a los injertos utilizados para el cierre de la duramadre (naturales o sintéticos). Esta revisión parece adecuada y oportuna, así como la discusión sobre las indicaciones de la operación. Desde la llegada de la RM se encuentran muchos indivi- guez Baeza, A., Cervera, C.: Posterior fossa reconstruction: a surgical technique for the treatment of Chiari I malformation and Chiari I/syringomyelia complex. Preliminary results and magnetic resonance imaging quantitative assessment of hindbrain migration. Neurosurgery 1994; 35: 874-885.

32. Sakamoto, H., Nishikawa, M., Hakuba, A., Yasui, T., Nakanishi, N., Inoue, Y.: Expansive suboccipital cranioplasty for the treatment of syringomyelia associated with Chiari malformation. Acta Neurochir (Wien) 1999; 141: 949-961.

33. Schijman, E., Steinbok, P.: International survey on the management of Chiari I malformation and syringomyelia. Childs Nerv Syst 2004; 20: 341-348.

34. Schmidek, H.H., Sweet, W.H.: Operative neurosurgical techniques: indication, methods and results. WB Saunders Company, Philadelphia 2000; pp. 1946-1954.

35. Vanaclocha, V., Saiz-Sapena, N., Garcia, M.: Surgical technique for craniocervical decompression in syringomyelia associated with Chiari type I malformation. Acta Neurochir (Wien) 1997; 139: 529-540

36. Weinberg, J.S., Freed, D.L, Sadock, J.: Headache and Chiari I malformation in the pediatric population. Pediatr Neurosurg 1998; 29:14-18.

37. Williams.: A blast against grafts on the closing and grafting of the posterior fossa dura. Br J Neurosurg 1994; 8: 275-278.

38. Won, D.J., Nambiar, U., Muszynski, C.A.: Coagulation of herniated cerebellar tonsils for cerebrospinal fluid pathway restoration. Pediatr Neurosurg 1997; 27: 272-275.

Saceda-Gutiérrez, J.M.; Isla-Guerrero, A.; Álvarez-Ruíz, F.; Odene-Cantero, C.; Hernández-García, B.; MárquezPérez, T.M.: Complicaciones postquirúrgicas de la malformación de Chiari tipo I: duroplastia y fístula de líquido cefalorraquideo. Neurocirugía 2011; 22: 36-43.

Correspondencia: Saceda Gutiérrez. Servicio de Neurocirugía. $2^{\text {a }}$ Planta Hospital General. Paseo de la Castellana no 261. 28046 Madrid.

Javisaceda@yahoo.es duos que tienen descenso de las amígdalas cerebelosas y que son remitidos a Neurocirugía. Lo más importante es sentar una indicación quirúrgica sobre bases sólidas y dejar para observación los casos dudosos u oligosintomáticos. En mi opinión la asociación de una siringomielia importante en niños o jóvenes representa una indicación de cirugía, ya que el daño medular producido por la siringomielia suele 
ser irreversible, y en estos pacientes la operación puede tener valor preventivo del deterioro de la función medular.

La aparición de fístulas de LCR y/o pseudomeningoceles supone un problema difícil de solucionar. Es muy importante, a la hora del cierre dural, comprobar la estanqueidad de la sutura realizando algunas maniobras de Valsaba por parte del anestesista, para detectar cualquier escape de liquido, aunque sea mínimo, volviendo a suturar las zonas dehiscentes, incluso con el microscopio quirúrgico. Además, se debe realizar un cierre muy apretado, incluso en varias capas, de los planos musculares. Si, a pesar de ello, aparece un pseudomeningocele nosotros realizamos alguna punción subcutánea del mismo, seguida de vendaje compresivo. Suelen bastar 1-2 punciones para resolver el lóculo. Además solemos administrar Edemox (200 mg 3 veces al día) durante un período de una a tres semanas. Caso de persistir la colección de LCR, realizamos una TAC para descartar la presencia de hidrocefalia y también consideramos la reexploración quirúrgica para cerrar la fístula.
Los autores mencionan de pasada un caso desafortunado de una fatalidad debida a la formación de un hematoma postoperatorio que experimentaron. Dado que cada vez se operan más pacientes con esta patología, quizás sería conveniente comentar aquí que estos enfermos deben ser vigilados estrechamente en la Reanimación postquirúrgica al menos la primera noche tras la operación. En la eventualidad de deterioro, que suele ser rápido y en las primeras horas, el paciente debe ser trasladado de inmediato a quirófano para reabrir la herida operatoria y descartar que se haya producido un hematoma. En mi opinión, esta exploración quirúrgica no debe demorarse, debiéndose realizar incluso antes de la obtención de una TAC urgente.

En resumen, el trabajo muestra la experiencia en el tratamiento de la anomalía de Chiari-1 en el Hospital La Paz de Madrid. Por último, felicitar a los autores por compartir honestamente sus resultados y sus conclusiones.

J.F. Martínez-Lage Murcia 